\title{
Nocturnal enuresis in a young woman treated with acupuncture and moxibustion: case report
}

\begin{abstract}
Nocturnal enuresis is intermittent involuntary emission of urine during sleep that does not awaken the patient. We present a 22-year-old woman with no known allergies or past medical history a part of a slight mental disability from childhood not affiliated. She suffers from occasional nocturnal enuresis especially when she is more relaxed at night and in menses period. Treatment with acupuncture and moxibustion improves this case in only four sessions of Acupuncture and self-Moxibustion at home. Acupuncture could be a safe and effective technique to improve nocturnal enuresis.
\end{abstract}

Keywords: nocturnal enuresis, acupuncture therapy, moxibustion
Volume 13 Issue 3 - 2020

\author{
Giralt Sampedro I,' Cayuela S, ${ }^{2}$ Nishishinya \\ Aquino $\mathrm{MB}^{3}$ \\ 'Medical Director Energimed,Woman Dexeus Hospital, Spain \\ ${ }^{2}$ Medical Acupuncturist at Energimed, Spain \\ ${ }^{3}$ Department of Rheumatology/Sports Medicine, Instituto \\ Traumatologia, Spain
}

Correspondence: Giralt Sampedro I, Medical Director Energimed, MD Acupuncturist at Woman Dexeus Hospital, Spain,Email isagiralt@gmail.com

Received: March 19, 2020 | Published: May 12, 2020

\section{Introduction}

Nocturnal enuresis is an involuntary emission of urine during sleep period that does not awaken the patient. It is a normal process in children that becomes pathological when it persists over 5years old. Nocturnal enuresis affects $15 \%$ of children over 5years of age, $10 \%$ of those over 10 years of age and $2 \%$ of those over 15 years of age.

Its pathophysiology is not yet fully understood and can lead to psychological and well-being problems for families and patients. ${ }^{1}$ Psychological and psychiatric problems aggravate and lengthen the resolution of the problem in many cases. ${ }^{2}$ Usual therapies to improve this clinical situation are varied: behavioral and habit changes, education, little fluid intake at night, exercises to improve bladder capacity, night alarm systems, pharmacological treatments (hormonal, antidepressant, anticholinergic, others), Para-Sacral transdermal nerve stimulation, functional magnetic stimulation, and complementary therapies as well.

Acupuncture is one of the most known complementary therapies with better efficacy in some pathologies, especially in pain. ${ }^{3,4} \mathrm{~A}$ systematic review published conclude that acupuncture and laser acupuncture may be more effective in reducing enuresis, although the evidence is weak. ${ }^{5}$ On the other hand, a review of 24 systematic reviews of the use of acupuncture in pediatrics, shows Acupuncture effective in five pathologies, including enuresis, and reports that only 6 of these reviews show adverse effects, none serious. A meta-analysis of 21 randomized studies with a total of 1590 patients conclude acupuncture is more effective than placebo or even pharmacological treatment to treat enuresis in childhood. However rigorous high quality RCTs are urgently needed.

\section{Case report}

We present a 22-year-old woman with no known allergies or past medical history a part of a slight mental disability from childhood not affiliated. She suffers from nocturnal enuresis especially when she is more relax at night and in menses period, about four times a week. Her daytime urination, more than six times a day, comes out with little force, as well. She suffers from lactose and fructose intolerance and often has abdominal distension after meals and morning diarrhea. She feels usually cold and she defines herself as a timid and fear person. She is not taken any medication.

\section{Examination}

Her phenotype is more a teenager like, she has only primary education, lives with her parents and works occasionally as a child monitor. She is a small girl and has pale skin and slightly overweight. On examination the tongue is small, flat and with dental marks and pulses are thin and weak.

\section{Treatment}

According to Chinese Medicine Kidney Qi Deficiency Syndrome is diagnosed: Kidney Qi deficiency is a cold syndrome that can occur with constitutional weakness and usually presents polyuria, nycturia and morning diarrhea, as well as a tendency to fear in the emotional sphere. The objective of our treatment is to tonify and warm the Kidney Qi, focusing in the urologic area. We apply four acupuncture sessions, one per week, the sessions lasted 20-minutes. Qi sensation was not sought, due to the high sensitivity of the patient. The selected points were: VC3 (Zhonji), ST29 (Guilai), KI3 (Taixi), P6 (Neiguan), ST36 (Zusanli), BL28 (Pangguangshu), BL67 (Zhiyin) and VG20 (Baihui). All points are bilateral points except those in VC and VG. Puncture depth has been $1.5 \mathrm{~cm}$. except at point DM20. We asked the patient to apply moxibustion. ${ }^{8,9}$ (application of heat with an artemisia cigar near the skin but not touching) in points VC3 (Zhonji), and BL67(Zhiyin) bilateral, every day for 5-10minutes, in total. The Acupuncture sessions was applied by two medical acupuncturists with more than 30years of experience Figure 1.

\section{Outcome}

After the second session, the patient reported a clear improvement, with more limited nighttime urination. From the third session, the enuresis has completely disappeared, the urine emerges strongly during daytime and the diarrheal episodes disappeared. In the psychological area her mood has also improved and she feels happier and less fear. We do a fourth acupuncture session confirming that she continues 
without enuresis. To maintain the results, we proposed apply daily moxibustion at home in points VC3 (Zhonji), and BL67(Zhiyin) even after the last acupuncture session. One month after the last session she told us that she has been free of enuresis after the third session except in one occasion during her menstruation. The patient did not report any side effects due to the treatments.
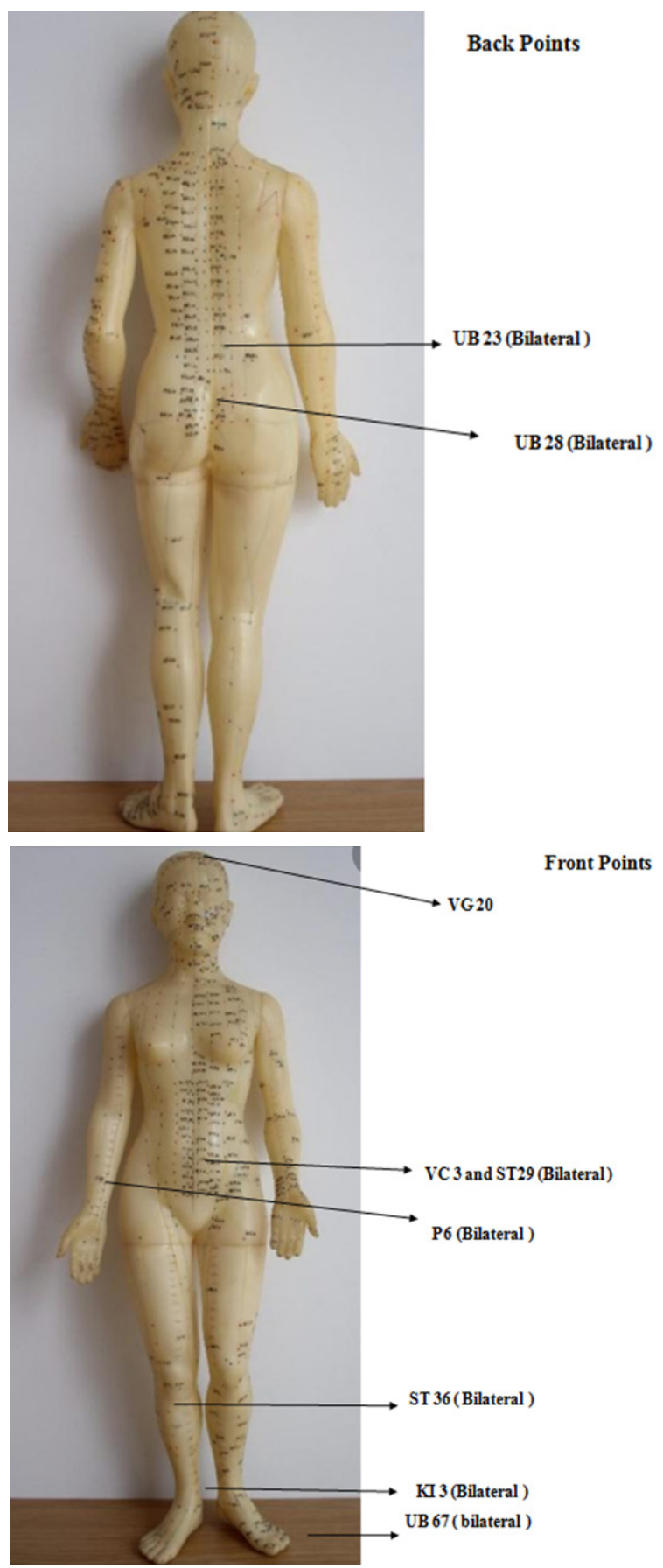

Figure I Location of stimulated acupuncture points on body.

\section{Discussion}

In this case we have been using points indicated in Kidney Qi Deficiency Syndrome: VC3, ST29, BL28, BL6, ST36, KI3 and, BL23. In our treatments, we add points VG20 and P6 because they are general regulatory points.
Moxibustion in VC3 and BL67 warm the interior and are useful for a Cold Syndrome in the Urinary System according to Traditional Chinese Medicine. ${ }^{8,9}$ Kidney Qi deficiency from Chinese Medicine Syndromes defines very well the pathology of this patient globally: mental disability, fearful and insecure person, urinary dysfunction, cold and the tendency to diarrea. ${ }^{9}$

Related to the bibliography the most common points to treat this disease are: BL 23, BL 28, BL 29. St 29, VC 3, VC 4. ${ }^{10,11}$ All of them related to the lumbosacral and hypogastric area. We can explain these points according to modern medicine, as well: We know bladder is innervated by the autonomic nervous system: parasympathetic roots from S2 to S4 and sympathetic roots from D10 to L2. The points areas we have been using in the lower half of the body are innervated by these roots. Some of the good results we obtained can be explained by the autonomic system regulation by Acupuncture..$^{12,13}$

\section{Conclusion}

With the experience limited to only one case but due to the highly positive results in only two months and after consulting the bibliography for the treatment of this pathology, we think that acupuncture and moxibustion, related to Traditional Chinese Medicine, can help to cure or ameliorate the Nocturnal Enuresis and the psychological problems which derives from it with non side effects. Some of the good results of the treatment could be explained in modern medicine for the Acupuncture effects in the Autonomic Nervous System, as well.

\section{Ethical responsibilities}

This is a case report from my private clinic thus ethical approval was not required.

\section{Data protection}

The authors declare that the patient has given consent to use her data for this article. Right to privacy and informed consent.

\section{Acknowledgments}

None.

\section{Conflicts of interest}

Declaration of competing interest Absence of any conflict of interest. We thank the patient for her collaboration and consent to publish this case.

\section{Funding}

None.

\section{References}

1. Van Herzeele C, Walle JV, Dhondt K, et al. Recent advances in managing and understanding enuresis. F1000Res. 2017;6:1881.

2. Nevéus T. Pathogenesis of enuresis: Towards a new understanding. Int $J$ Urol. 2017;24(3):174-182.

3. Vickers AJ, Cronin AM, Maschino AC, et al. Individual patient data metaanalysis of acupuncture for chronic pain: protocol of the Acupuncture Trialists' Collaboration. Acupuncture Trialists' Collaboration. Trials. 2010;11:90.

4. Vickers AJ, Vertosick EA, Lewith G, et al. Acupuncture Trialists' Collaboration. Acupuncture for Chronic Pain: Update of an Individual Patient Data Meta-Analysis. J Pain. 2018;19(5):455-474. 
5. Kiddoo D. Nocturnal enuresis: non-pharmacological treatments. $B M J$ Clin Evid. 2015;2015. pii: 0305.

6. Yang C, Hao Z, Zhang LL, et al. Efficacy and safety of acupuncture in children: an overview of systematic reviews. Pediatr Res. 2015;78(2):112119.

7. Zhu J, Arsovska B, Kozovska K. Nocturnal enuresis in children Treatment with acupuncture. Journal of Dental and Medical Sciences (IOSR-JDMS). 2016;15(19):29-31.

8. Radvanska E, Kamperis K, Kleif A, et al. Effect of Laser Acupuncture for Monosymptomatic Nocturnal Enuresis on Bladder Reservoir Function and Nocturnal Urin. J Urol. 2011;185(5):1857-1861.

9. Lv ZT, Song W, Wu J, et al. Efficacy of Acupuncture in Children with Nocturnal Enuresis: A Systematic Review and Meta-Analysis of Randomized Controlled Trials. Evid Based Complement Alternat Med. 2015;2015:320701.
10. Carl-Hermanna Hempem, Velia Wortman Chow. Pocket Atlas of Acupuncture. Germany: Thieme Stuttgart; 2015. ISBNISBN3-13-141721-8.

11. Hans Ulrich Hecker. Practice of Acupuncture. New York, USA: Thieme, Stettgart; ISBN 3-13-136821-7.

12. Linda Wilson Pauwels, Patricia A Stewart, Elizabeth J Akesson. Autonomic Nerves. Canada: PMPH USA; 2015. ISBN 1-55009-030-5.

13. Jaqueline Filshie, Adrian White. Medical Acupuncture. England: Churchill Livingstone; ISBN $0-443-04976-9$. 